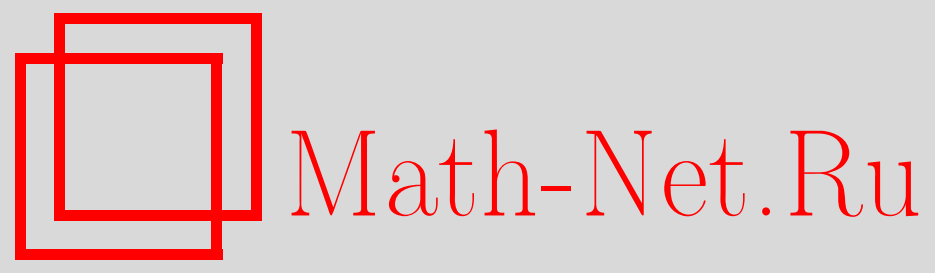

K. К. Щукин, О примитивных подгруппах полной аффинной группы конечного почти-поля, Дискрет. матем., 2003, том 15, выпуск 4, 126-132

DOI: https://doi.org/10.4213/dm221

Использование Общероссийского математического портала Math-Net.Ru подразумевает, что вы прочитали и согласны с пользовательским соглашением http: //www . mathnet.ru/rus/agreement

Параметры загрузки:

IP : 54.92 .164 .108

26 апреля 2023 г., 06:41:13 


\title{
О примитивных подгруппах полной аффинной группы конечного почти-поля
}

\author{
() 2003 г. К. К. Щукин
}

\begin{abstract}
В этой работе завершается начатое автором ранее изучение конечных примитивных групп, стабилизатор точки которых содержит абелеву нормальную подгруппу, действующую неприводимо (сопряжениями) на абелевой нормальной подгруппе всей группы. Каждая такая группа $H$ изоморфна подгруппе $Z_{p}^{\nu} \lambda \Theta \lambda \Psi$ полной аффинной группы $A\left(F_{p^{\nu}}\right) \cong Z_{p}^{v} \lambda Z_{p^{\nu}-1} \lambda Z_{p}$ поля $F_{p^{\nu}}$, где знаком полупрямого произведения $\lambda$ соединены $\nu$-степень циклической группы $Z_{p}$, метациклическая группа $\Theta$ и (некоторая) группа автоморфизмов $\Psi$ поля $F_{p^{\nu}}$. С использованием классификации конечных почти-полей Цассенхауза, перечисляются и примитивные подгруппы полных аффинных групп конечных почти-полей.
\end{abstract}

1. В этой работе завершается начатое автором в [1-4] изучение конечных примитивных групп, стабилизатор точки которых содержит абелеву нормальную подгруппу, действующую неприводимо (сопряжениями) на абелевой нормальной подгруппе всей группы.

Для леводистрибутивного почти-поля $F=(F,+, \cdot)$ пусть Aut $F$ обозначает полную группу его автоморфизмов, $\operatorname{Aut}(F,+)$ - группу автоморфизмов его аддитивной группы $(F,+)$. Если $G=(G, \cdot)$ - подгруппа его мультипликативной группы $F^{*}=\left(F^{*}, \cdot\right)$, то $\Lambda(G, \cdot)=\Lambda(G)$ будет обозначать ее образ в левом регулярном представлении

$$
\lambda F^{*}: \rightarrow S(F), \quad a \rightarrow \lambda_{a}, \quad \lambda_{a}(x)=a x, \quad a, x \in F, \quad a \neq 0,
$$

групшы $F^{*}$ в симметрической группе $S(F)$. Для каждой подгруппы $\Psi$ группы Aut $F$ имеет смысл говорить о ее действии на $\Lambda(G)$ сопряжением, то есть о полупрямом произведении $\Lambda(G) \lambda \Psi$, если $G$ является $\Psi$-допустимой, то есть если

$$
\psi \lambda_{g} \psi^{-1}=\lambda \psi(a), \quad g \in G, \quad \psi \in \Psi
$$

Эти и другие подгруппы в группе

$$
\Lambda_{F}=\Lambda\left(F^{*}\right) \lambda \text { Aut } F
$$

и являются предметом нашего начального изучения, в основном для конечного случая.

Предложение 1. Пусть $F-$ поле и $\Phi-$ подгруппа группы Aut $(F,+)$, coдержащая неприводимую абелеву подгруппу х в качестве своей нормальной подгруппы. Тогда

(1) $\chi$ сопряжена в $\operatorname{Aut}(F,+)$ с подгруппой $\Gamma$ группы $\Lambda\left(F^{*}\right)$; 
(2) нормализатор Г в $\operatorname{Aut}(F,+)$ совпадает $c \Lambda_{F}=\Lambda\left(F^{*}\right) \lambda$ Aut $F$;

(3) Ф сопряжена в $\operatorname{Aut}(F,+)$ с подгруппой группы $\Lambda_{F}$.

Доказательство см. в [1], предложения 1, 2, и в [2], теорема 3.10.

Предложение 2. Для подгруппы $G$ группы $F^{*}$ конечного поля $F=F_{p}^{\nu}$, где $p$ - простое число, следующие условия эквивалентны:

(1) кольчо, натянутое на $G$ в $F$, совпадает с полем $F$;

(2) порядок $p(\bmod |G|)=v$;

(3) $\Lambda(G)$ неприводимо действует на группе $(F,+)$;

(4) $\Lambda(G) \lambda \Psi$ неприводимо действует на группе $(F,+)$ для любой группы автоморфизмов $\Psi$ поля $F$.

Доказательство см. в [1], лемма 1.

Замечание 1. Известно [3] следующее утверждение: для каждого простого $p$ и натурального числа $v$ существует такое простое число $p_{0}$, что порядок $p_{0}(\bmod p)=v$, за исключением случаев, когда $v=2, p$ - простое число Ферма и $v=6, p=2$. Так что за этими исключениями, согласно пункту (1) предложения 2 , в группе $\Lambda\left(F^{*}\right), F=F_{p^{\nu}}$, всегда существует неприводимая подгруппа простого порядка $p_{0}$.

Напомним еще (см. [4-6]), что класс $D F(q, n)$ конечных почти-полей Диксона из $q^{n}$ элементов, где $q=p^{n}, p$ - простое число, не пуст, если и только если $q, n-$ диксонова пара чисел, то есть если и только если каждое простое $r$ или $r=4$, которое делит $n$, должно делить и $q-1$. У всякого $F_{q, n} \in D F(q, n)$ его мультипликативная группа изоморфна метациклической группе $G_{q, n}$ специального вида. Отсюда, поскольку $F_{q, n}$ и спаренное с ним поле $F=F_{q}$ имеют общую аддитивную группу $(F,+)$, группа $\Lambda\left(F_{q, n}^{*}\right)$ сопряжена в $\operatorname{Aut}(F,+)$ с подгруппой группы $\Lambda_{F}$ поля $F_{q, n}$. Уточнение этого факта содержит следующая теорема.

Теорема 1. Пусть $F=F_{p^{v}}-$ поле, $p-$ простое число,

$$
\Lambda\left(F^{*}\right)=\left\langle\lambda_{a}\right\rangle, \quad \text { Aut } F=\langle\eta\rangle .
$$

Тогда каждая подгруппа $\Theta$ группь

$$
\Lambda_{F}=\Lambda\left(F^{*}\right) \lambda \text { Aut } F
$$

с нормальной в $\Theta$ абелевой подгруппой Г порядка $m$, неприводимо действующей на группе $(F,+)$, будучи метачиклической, в общем случае имеет порядок $m$, где пи $=v, u$ представление

$$
\Theta \cong\left\langle x y \mid x^{m}=1, y^{n}=x^{l}, y x y^{-1}=x^{q}\right\rangle,
$$

где $q=p^{n}$, которое осуществляется, когда

$$
\gamma=\lambda_{a}^{k}, \quad \Gamma=\langle\gamma\rangle, \quad \omega=\lambda_{a}^{s} \eta^{n}, \quad \Theta / \Gamma=\langle\Gamma \omega\rangle,
$$

при этом для порядка q справедливы соотношения

$$
q \quad(\bmod m)=n, \quad q-1=0 \quad(\bmod m), \quad(m, q)=1 .
$$


Доказательство. Если подгруппа $\Theta$ нециклическая, $\Gamma=\langle\gamma\rangle, \gamma=\lambda_{a}^{k}$, имеет порядок $m$, а $\Theta / \Gamma=\langle\Gamma \omega\rangle-$ порядок $n$ и $m n \mid p^{\nu}-1$, то $\omega \lambda_{a} \neq \lambda_{a} \omega$, то есть

$$
\omega=\lambda_{a}^{s} \psi, \quad 0 \leqslant s<p^{\nu}-1, \quad \psi \in\langle\eta\rangle, \quad \psi \neq 1
$$

Так как

$$
\omega^{i}=\lambda_{a+\psi(a)+\ldots+\psi^{i}(a)}^{s} \psi^{i}, \quad i=0,1, \ldots,
$$

из предположения, что $\omega^{n} \in \Gamma$, следует, во-первых, что $\psi^{n}=1$, то есть $\psi=\eta^{u}$, где $u=v / n,-$ автоморфизм Фробениуса поля $F_{q^{n}}$ над полем $F_{q}$, где $q=p^{n}, \psi(a)=a^{q}$. Во-вторых,

$$
\omega^{n}=\lambda_{a}^{s\left(1+q+\ldots+q^{n}\right)} \psi^{n}=\lambda_{a}^{s\left(q^{n}-1\right) /(q-1)}=\lambda_{a}^{k l}
$$

для некоторого целого числа $l$. Полагая $m^{\prime}=\left(q^{n}-1\right) / m$, запишем $k=k^{\prime} m^{\prime}$, где $\left(k^{\prime}, m^{\prime}\right)=1$. Отсюда, равенство $s\left(q^{n}-1\right)=(q-1) k l$ равносильно равенству $s m=(q-1) k^{\prime} l$, то есть

$$
(q-1) l \equiv 0 \quad(\bmod m)
$$

Далее,

$$
\omega \gamma \omega^{-1}=\omega \lambda_{a}^{k} \omega^{-1}=\lambda_{a}^{k q}=\gamma^{q} .
$$

Понятно, что $(q, m)=1$, и остается напомнить, что сравнение $q(\bmod m)=n$ следует из предположения о неприводимости $\Gamma$, согласно предложению 2.

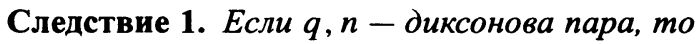

$$
\Theta \cong G_{q, n},
$$

причем

$$
|\Theta|=m n=q^{n-1}, \quad(q-1) l=m .
$$

Более того, подгруппа $\Theta$ эквивалентна $\Lambda\left(F_{q, n}\right)$ на $F$ для некоторого $F_{q, n} \in D F(q, n)$, спаренного с полем $F=F_{q^{n}}$.

Доказательство. Пусть $F=F_{q^{n}}$ и $F_{q, n}=(F,+$, o) - спаренное с ним почти-полем Диксона, тогда умножение в нем $x$ о $y$ для $x, y \in F$ задается формулой (см. [5])

$$
x \circ y= \begin{cases}x \psi^{i}(y), & \text { если } \lambda_{x} \in \Gamma \lambda_{a}^{\left(q^{i}-1\right) /(q-1)}, i=1,2, \ldots, n, \\ 0, & \text { если } x=0,\end{cases}
$$

в силу разложения

$$
\Lambda\left(F^{*}\right)=\bigcup_{i=1}^{n} \Gamma \lambda_{a}^{\left(q^{i}-1\right) /(q-1)}
$$

справедливого для диксоновой пары $q, n$ при $m=(q-1) / n$. Но в этом случае

$$
\Theta=\bigcup_{i=1}^{n} \Gamma \lambda_{a}^{\left(q^{i}-1\right) /(q-1)} \psi^{i}
$$


и ввиду биекции

$$
\Gamma_{a}^{\left(q^{i}-1\right) / q-1} \rightarrow \Gamma \lambda_{a}^{\left(q^{i}-1\right) /(q-1)} \psi^{i}, \quad i=1,2, \ldots, n
$$

получаем, что для $x \in F, x \neq 0$, действие $\Theta$ на $F$ задается равенством

$$
\lambda_{x} \psi^{i}(y)=\lambda_{x} \psi^{i}(y)=x \circ y,
$$

если $\lambda_{x} \in \Gamma \lambda_{a}^{\left(q^{i}-1\right) /(q-1)}$.

Заметим дополнительно, что число неэквивалентных подгрупп $\Theta \cong G_{q, n}$ в $\Lambda_{F}$ для данной диксоновой пары $q, n$ равно, очевидно, $\varphi(n)$, где $\varphi(n)-$ функция Эйлера. В этой связи уместно напомнить так же следующее утверждение из [1].

Предложение 3. В условиях и обозначениях теоремы 1 имеют место следующие утверждения:

(1) число $\alpha\left(p^{\nu}-1\right)$ неизоморфных подгрупп $\Gamma \cong Z_{m}$ в группе $\Lambda\left(F^{*}\right)$ таких, что порядок $p(\bmod m)=\nu$, вычисляется по формуле

$$
\alpha\left(p^{\nu}-1\right)=\sum_{d \mid \nu} \mu(d) \tau\left(p^{\nu / d}-1\right)
$$

где $\mu(s), \tau(s)$ - это, соответственно, функция Мебиуса и число чельх положительных делителей числа $s \in \mathbf{N}$; если же $v=r s$ для некоторого простого числа $r$, то справедлива и формула

$$
\alpha\left(p^{\nu}-1\right)=\tau\left(p^{\nu}-1\right)-\tau\left(p^{\nu / d}-1\right),
$$

(2) число $\beta\left(p^{\nu}-1\right)$ неизоморфных подгрупп вида $\Gamma 入 \Psi$ в группе

$$
\Lambda_{F}^{*}=\Lambda\left(F^{*}\right) \lambda \Psi \cong Z_{m} \lambda Z_{d},
$$

где $\Psi \leqslant$ Aut $F, m, d$ те же, что и в пункте (1), но $d>1$, дает формула

$$
\beta\left(p^{\nu}-1\right)=(\tau(\nu)-1) \alpha\left(p^{\nu}-1\right) .
$$

Ясно, что все группы вида $\Gamma \lambda \Psi$ относятся к $Z$-метациклическим, для которых в общем случае выполняется дополнительное условие $(m, q-1)=1$, что и дает $y^{v}=1$ в их представлении. В нашем случае это означает, что

$$
\lambda_{a}^{s\left(q^{n}-1\right) /(q-1)}=1
$$

или

$$
s\left(q^{\nu}-1\right)=(q-1) k^{\prime} l^{\prime} m, \quad s m m^{\prime}=(q-1) k m m^{\prime},
$$

откуда, $s \equiv 0(\bmod q-1)$, а значит, $y=\psi$, и мы получаем следующее дополнение к утверждению теоремы 1 : в группе $\Lambda_{F}$ единственными $Z$-метациклическими подгруппами являются группы вида $\Gamma \lambda \Psi$ из пункта 2 предложения 3 . 
2. Напомним некоторые факты (см. $[5,8])$. Прежде всего, если симметрическая группа $S(F)$ содержит регулярную подгруппу $T$, то на множестве $F$ можно ввести структуру аддитивной группы $(F,+)$ с нулем 0 так, что $T=T(F,+)$ является образом $(F,+)$ при ее (левом) регулярном представлении

$$
t: F \rightarrow S(F), \quad t(x)=t_{x}, \quad t(x+y)=t_{x} t_{y}, \quad t_{x} t_{0}=t_{x}=t_{0} t_{x}, \quad x, y, 0 \in F, \quad t_{x} \in T t .
$$

Если к тому же $\operatorname{Aut}(F,+)$ содержит подгруппу $\Gamma$, регулярно действующую на множестве

$$
F^{*}=F-\{0\},
$$

то это позволяет так определить умножение на $F$ в $(F, \cdot)$, что образуется структура почтиполя $F=(F,+, \cdot)$ с нулем и единицей 1 . При этом $(F,+)$ оказывается с необходимостью абелевой группой, и $\Lambda=\Lambda\left(F^{*}\right)$ будет образом мультипликативной группы $\left(F^{*}, \cdot\right)$ при ее (левом) регулярном представлении

$$
\lambda: F^{*} \rightarrow \Lambda, \quad \lambda(a)=\lambda_{a}, \quad \lambda_{a}(x)=a x, \quad a, x \in F, \quad a \neq 0 .
$$

Определение 1. Аффинной группой $A(F)$ почти-поля $F=(F,+, \cdot)$ назовем подгруппу

$$
A(F)=T(F,+) \lambda \Lambda\left(F^{*}\right)
$$

голоморфа

$$
\operatorname{Hol}(F,+)=T(F,+) \lambda \operatorname{Aut}(F,+)
$$

его аддитивной группы $(F,+)$, а его полная аффинная группа $\tilde{A}(F)$ определяется полупрямым произведением

$$
\tilde{A}(F)=A(F) \lambda \operatorname{Aut}(F) .
$$

Отметим, что для каждого почти-поля $F=(F,+, \cdot)$ имеют место следующие утверждения [5].

Для $\Phi \leqslant$ Aut $F$ и $\Phi$-допустимой $\Gamma \leqslant \Lambda\left(F^{*}\right)$ справедливо равенство

$$
(T(F,+) \lambda \Gamma) \lambda \Phi=T(F,+) \lambda(\Gamma \lambda \Phi)
$$

с учетом действия $\Phi$ на $\Lambda\left(F^{*}\right)$ сопряжениями

$$
\varphi \lambda_{a} \varphi^{-1}=\lambda_{\varphi(a)}, \quad \varphi \in \Phi, \quad \lambda_{a} \in \Lambda\left(F^{*}\right),
$$

при этом

$$
H=T(F,+) \lambda \Gamma \lambda \Phi
$$

примитивна на множестве $F$ тогда и только тогда, когда $\Gamma$ неприводима как подгруппа $\operatorname{Aut}(F,+)$.

$\Lambda\left(F^{*}\right) \lambda$ Aut $F$ совпадает с нормализатором $\Lambda\left(F^{*}\right)$ в $\operatorname{Aut}(F,+)$.

Аффинные группы двух изоморфных почти-полей с одной и той же аддитивной группой $(F,+)$ сопряжены в $\operatorname{Hol}(F,+)$.

Кроме того, справедливо следующее утверждение. 
Предложение 4. Пусть примитивная подгруппа $H$ и абелева транзитивная подгруппа $T$ в $S(F)$ таковы, что $T \triangleleft H u$

$$
H_{0}=\left\{h \in H \mid h t_{0} h^{-1}=t_{0}\right\} .
$$

Тогда

(1) если $H_{0}$ содержит нормальную абелеву подгруппу $\Gamma$, действуюшую неприводимо на $T$ сопряжениями, то $H$ является подгруппой полной аффинной группь $\tilde{A}(F)$ поля $F=(F,+, \cdot) \operatorname{ct}(F,+)=T, \lambda\left(F^{*}, \cdot\right)=\Lambda$;

(2) если $H_{0}$ содержит нормальную подгруппу $\Lambda$, действуюшую регулярно на $T^{*}=T \backslash t_{0}$, то $H$ - подгруппа полной аффинной группы $\tilde{A}(F)$ почти-поля $F=(F,+, \cdot) c$ $t(F,+)=T, \Lambda\left(F^{*}, \cdot\right)=\Lambda$.

В конечном случае версия последнего предложения принимает вполне законченный вид, если учесть, что мощность каждого конечного почти-поля равна примарной степени.

Ниже везде $Z_{s}^{r}$ обозначает прямую степень циклической группы $Z_{s}$.

Теорема 2. Пусть $F=(F,+, \cdot)-$ конечное почти-поле из $p^{\nu}$ элементов,

$$
\tilde{A}(F)=T(F,+) \lambda \Lambda\left(F^{*}\right) 入 \text { Aut } F
$$

- его полная аффинная группа,

$$
H=T(F,+) \lambda H_{0}
$$

- ее примитивная подгруппа,

$$
H_{0}=\left\{h \in H \mid h t_{0} h^{-1}=t_{0}\right\} .
$$

Тогда, с точностью до сопряжения, в $\tilde{A}(F)$ существуют только следующие группы типа $H \cong Z_{p}^{v} \lambda H_{0}$.

(I) Разрешимые группы.

(A) $H_{0}$ содержит свою абелеву нормальную подгруппу Г порядка $m$, действующую неприводимо на $T(F,+)$ сопряжениями. Тогда

(1) $F=G F\left(p^{\nu}\right), H \cong Z_{p}^{n} \lambda \Theta \lambda \Psi$, где $\Theta-$ метачиклическая группа порядка $m n, v / n=u$, из теоремы 1, в частности, $\Theta \cong G_{q, n}$ в случае диксоновой nары $q=p^{u}, n u \Psi \leqslant$ Aut $F$;

(2) $F \in D F(3,2), H \cong Z_{3}^{2} \lambda Q_{8} \lambda S_{3}$, где $Q_{8}, S_{3}-$ группа кватернионов $и$ симметрическая группа, соответственно;

(В) $H_{0}$ не содержит абелевой нормальной подгруппы, действующей неприводимо на $T(F,+)$. Тогда $F$ - (некоммутативное) почти-поле и (для максимальной $H)$ возможны только случаи

(3) $|F|=5^{2}, \tilde{A}(F) \cong Z_{5}^{2} \lambda T^{*} \lambda Z_{4}, T^{*} \cong S L_{2}(3)$;

(4) $|F|=11^{2}, \tilde{A}(F) \cong Z_{11}^{2} \lambda\left(T^{*} \lambda Z_{5}\right) \lambda Z_{2}$;

(5) $|F|=7^{2}, \tilde{A}(F) \cong Z_{7}^{2} \lambda O^{*} \lambda Z_{3}$;

(6) $|F|=23^{2}, \tilde{A}(F) \cong Z_{23}^{2} \lambda\left(O^{*} \lambda Z_{11}\right.$. 
(II) Неразрешимые группы (для максимальной $H$ ):

(7) $|F|=11^{2}, \tilde{A}(F) \cong Z_{11}^{2} \lambda I^{*} \lambda Z_{5}, I^{*} \cong S L_{2}(5)$;

(8) $|F|=29^{2}, \tilde{A}(F) \cong Z_{29}^{2} \lambda\left(I^{*} \lambda Z_{7}\right) \lambda Z_{2}$;

(9) $|F|=59^{2}, \tilde{A}(F) \cong Z_{59}^{2} \lambda\left(I^{*} \lambda Z_{29}\right)$.

Заметим, что группы $T^{*}, O^{*}, I^{*}$ называют, соответственно, бинарными группами тетраэдра, октаэдра и икосаэдра. Все они являются расширениями своего центра $Z \cong Z_{2}$, который не выделяется прямым множителем $T^{*} / Z \cong A_{4}, O^{*} / Z \cong S_{4}, I^{*} / Z \cong A_{5}$. Их подгруппы хорошо известны, и подробно о них см. в приложениях $J$ и $I$ монографии [8].

\section{Список литературы}

1. Щукин К. К., О примитивных разрешимых группах перестановок с коммутативным стабилизатором точки. Сиб. матем. ж. (1993) 34, №3, 214-217.

2. Щукин К. К., О примитивных разрешимых группах перестановок с коммутативным стабилизатором точки. Изв. АН Республики Молдова, сер. матем. (1994), №2(15), 40-50.

3. Щукин К. К., Неприводимые абелевы группы автоморфизмов абелевой группы и примитивные разрешимые группы перестановок. Изв. РАН, сер. матем. (1994) 58, №2, 189-195.

4. Щукин К. К., Письмо в редакцию. Сиб. матем. ж. (1996) 60, №6, 222.

5. Zassenhaus H., Über endliche Fastkörper. Abh. Math. Semin. Hamburg Univ. (1935) 11, 187-220.

6. Холл М., Теория групn. ИЛ, Москва, 1963.

7. Wahling H., Theorie der Fastkörper. Thales Verlag, Berlin, 1987.

8. Huppert B., Endliche Gruppen. Bd. I. Springer, Berlin, 1967.

9. Birkhoff G. D., Vandiver H. S., On the integral divisor of $a^{n}-b^{n}$. Ann. Math. (1904) 5, 173-180.

10. Супруненко Д. А., Группы матрич. Наука, Москва, 1972.

Статья поступила 03.01.2001. 